\title{
A Patient With Late-onset Limb-girdle Muscular Dystrophy Type 2B Mimicking Dermatomyositis: A Case Report and Review
}

\author{
Min Jung Kim, M.D. ${ }^{1}$, Yoon-Jeong Oh, M.D. ${ }^{2}$, Yoon Ho Hong, M.D., Ph.D. ${ }^{3}$, Sung-Hye Park, M.D., Ph.D. ${ }^{\text {, }}$ \\ Ji Seon Oh, M.D., Ph.D. ${ }^{5}$, Min Jung Kim, M.S. ${ }^{6}$, Jong-Hee Chae, M.D., Ph.D. ${ }^{6}$, Kichul Shin, M.D., Ph.D. ${ }^{1}$ \\ ${ }^{1}$ Division of Rheumatology, Seoul Metropolitan Government-Seoul National University Boramae Medical Center, Seoul, ${ }^{2}$ Division of \\ Rheumatology, Department of Internal Medicine, Kangwon National University School of Medicine, Chuncheon, ${ }^{3}$ Department of Neurology, \\ Seoul Metropolitan Government-Seoul National University Boramae Medical Center, ${ }^{4}$ Department of Pathology, Seoul National University \\ College of Medicine, ${ }^{5}$ Clinical Research Center, Asan Medical Center, University of Ulsan College of Medicine, ${ }^{6}$ Department of Pediatrics, Seoul \\ National University Children's Hospital, Seoul National University College of Medicine, Seoul, Korea
}

Limb-Girdle Muscular Dystrophy 2B (LGMD2B) presents with proximal and/or distal muscle weakness and markedly high creatine kinase level. It is caused by the loss of dysferlin due to mutations in the DYSF gene. Due to its similar clinical features as inflammatory myopathy, it is often difficult to distinguish between the two. We present a case of a 48-year-old male who developed progressive proximal muscle weakness, papulosquamous lesions on the knuckles, elevated levels of muscle enzymes, and electromyogram abnormalities. Based on the clinical presentation, the initial impression was dermatomyositis, yet it was refractory to immunosuppressive therapy. Subsequently, dysferlin immunostaining and genetic analysis led to the final diagnosis of LGMD2B. This case shows that LGMD2B can present with extramuscular symptoms mimicking inflammatory myopathy in later stages of life. Dysferlin immunostaining and/or genetic analysis of the DYSF gene are essential for its diagnosis. (J Rheum Dis 2021;28:101-106)

Key Words. Muscular dystrophies, Limb-girdle, Dermatomyositis

\section{INTRODUCTION}

Inflammatory myopathy is a chronic inflammatory muscle disease caused by immune-mediated muscle injury and consists of dermatomyositis (DM), polymyositis (PM), inclusion body myositis and immune-mediated necrotizing myopathy (IMNM). Patients with DM or PM present with progressive proximal muscle weakness over weeks to months and show good responses to immunosuppressive therapy [1]. DM is associated with distinct skin manifestations such as Gottron's papules or a heliotrope eruption, which are useful in distinguishing between inflammatory myopathies. Additionally, other muscle diseases such as muscular dystrophy, metabolic, endocrine or drug-induced myopathies may mimic inflammatory myopathy, but none of these disorders are associated with the skin lesions characteristic of DM [2].

Limb-girdle muscular dystrophy 2B (LGMD2B), a subtype of dysferlinopathy, is characterized by predominantly proximal muscle weakness and markedly high creatine kinase (CK) levels [3]. LGMD2B, caused by the dysferlin gene (DYSF) mutation, usually occurs in childhood or early adolescence [4]. It may present as similar as inflammatory myopathy in terms of clinical, laboratory, and electromyography (EMG) features. Histologically, inflammatory cell infiltrations in myofibers could be present in LGMD2B [5]. Therefore, a late-onset presentation of LGMD2B can at times be mistaken for inflammatory

Received : October 19, 2020, Revised : November 22, 2020, Accepted : November 25, 2020

Corresponding to : Kichul Shin (D http://orcid.org/0000-0002-6749-7598

Division of Rheumatology, Seoul Metropolitan Government-Seoul National University Boramae Medical Center, 20 Boramae-ro 5-gil, Dongjak-gu, Seoul 07061, Korea. E-mail : kideb1@snu.ac.kr 
myopathy.

Herein we report a case of a 48 -year-old male with progressive proximal muscle weakness, papulosquamous lesions in the knuckles, elevated CK levels, and myopathic changes on the EMG. The initial impression of his diagnosis was DM, yet the final diagnosis turned out to be LGMD2B with psoriasis.

\section{CASE REPORT}

A 48-year-old male presented with proximal muscle weakness in his lower extremities followed by skin rashes on the hands. Muscle weakness had slowly progressed over the past eight months. He had difficulty in sitting up and could only move around using a wheelchair or a walker. He denied myalgia, arthralgia, or dysphagia. He was diagnosed with type 2 diabetes mellitus, hypertension, and dyslipidemia during the past three years. He had a second degree burn over $70 \%$ of his face ten years ago. He was a 15-pack-year smoker with no occupation and drank alcohol once a week. He was taking aspirin, atorvastatin, perindopril and nateglinide. His family history of medical illness was unremarkable.

On physical examination, his vital signs were stable, and the body mass index was $19.8 \mathrm{~kg} / \mathrm{m}^{2}$ (height $165 \mathrm{~cm}$, body weight $54 \mathrm{~kg}$ ). Proximal muscle strength in both lower extremities was grade 3 out of 5 based on the Medical Research Council scale. The strength in his distal lower extremities and the proximal and distal upper extremities was grade $4+$. Calf muscle atrophy was not observed. Erythematous papulosquamous skin lesions with scales were present on the dorsal surface of his metacarpophalangeal joints, fingers, and elbows. Physical findings in other systems and organs were unremarkable. At his first visit, CK level was 4,512 IU/L (normal 45 163), aldolase was $18.2 \mathrm{U} / \mathrm{L}(0 \sim 7.6)$, and lactate dehydrogenase was $432 \mathrm{IU} / \mathrm{L}(100 \sim 225)$. Aspartate transaminase and alanine transaminase levels increased to $75 \mathrm{IU} / \mathrm{L}$ (1 40) and $77 \mathrm{IU} / \mathrm{L}(1 \sim 40)$, respectively. His complete blood count was as follows: white blood cell count 6,220 $/ \mu \mathrm{L}$, hemoglobin $14.7 \mathrm{~g} / \mathrm{dL}$, and platelets $267 \times 10^{3} / \mu \mathrm{L}$. The erythrocyte sedimentation rate and C-reactive protein levels were $8 \mathrm{~mm} / \mathrm{hr}(0 \sim 9)$ and $1.57 \mathrm{mg} / \mathrm{dL}(0 \sim$ $0.5)$, respectively. Anti-nuclear antibody, rheumatoid factor, and anti-Jo-1 antibody were not detected. EMG revealed small-amplitude, short-duration and polyphasic motor-unit potentials without increased spontaneous activity in the deltoid, biceps brachii, and pronator teres, and high amplitude and long duration motor-unit potentials in the vastus lateralis, tibialis anterior, and gastrocnemius muscles. Nerve conduction studies were normal. Results of the electrocardiogram and chest radiograph were unremarkable. There was no evidence of malignancy on further evaluation. For economic reasons, muscle imaging or biopsy was not initially performed.

With the suspicion of DM (symmetric proximal muscle weakness, scaly patchy redness over knuckles, elevated muscle enzyme levels, and EMG abnormalities), 20 $\mathrm{mg} /$ day of prednisolone and $300 \mathrm{mg} /$ day of hydroxychloroquine were given. However, muscle weakness did not respond to the initial combination of medications within 6 months. Erythematous papules and plaques with silvery scales on his elbows, hands, and legs were confirmed as psoriasis by a Dermatologist; topical ointment and $12.5 \mathrm{mg} /$ week of methotrexate were prescribed. Despite the immunosuppressive treatment, muscle weakness continued to worsen and CK levels fluctuated throughout the following 12 months (Figure 1). Eventually, a muscle biopsy of the vastus lateralis was performed. The specimen showed muscle cell degeneration, as well as regeneration and atrophy. Fatty replacement of muscle tissue was severe. There were no muscle cell necrosis or inflammatory cell infiltrates in endomysial and perivascular regions. Neural cell adhesion molecule (CD56) and dystrophin were present, yet dysferlin was absent on immunostaining (Figure 2). Electron microscopy did not show any tubuloreticular inclusion (Figure 3). Furthermore, a molecular analysis with Next Generation Sequencing disclosed heterozygous mutations in the DYSF gene; c.2548C $>$ T (p.Gln850*) and c.3051G > T (p.Trp1017Cys)

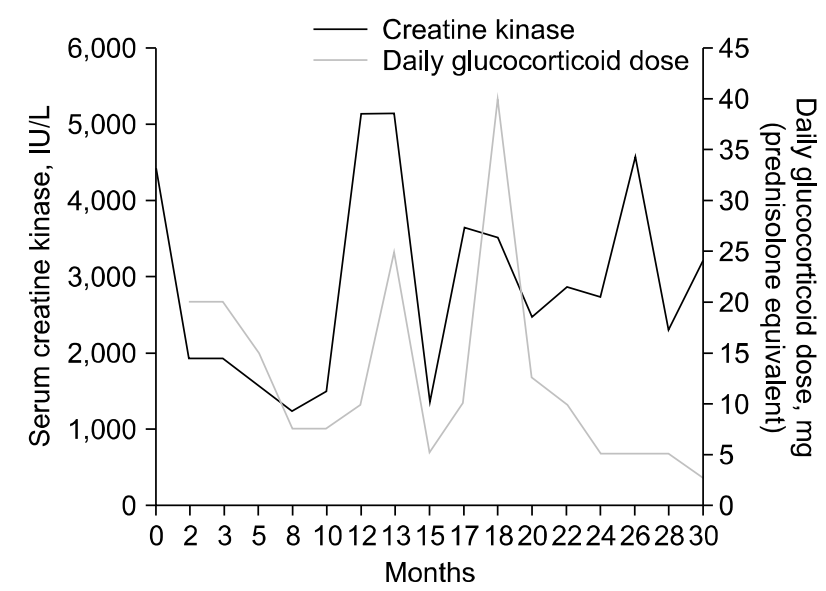

Figure 1. Changes in serum creatine kinase levels and daily doses of glucocorticoid during the follow-up period. 


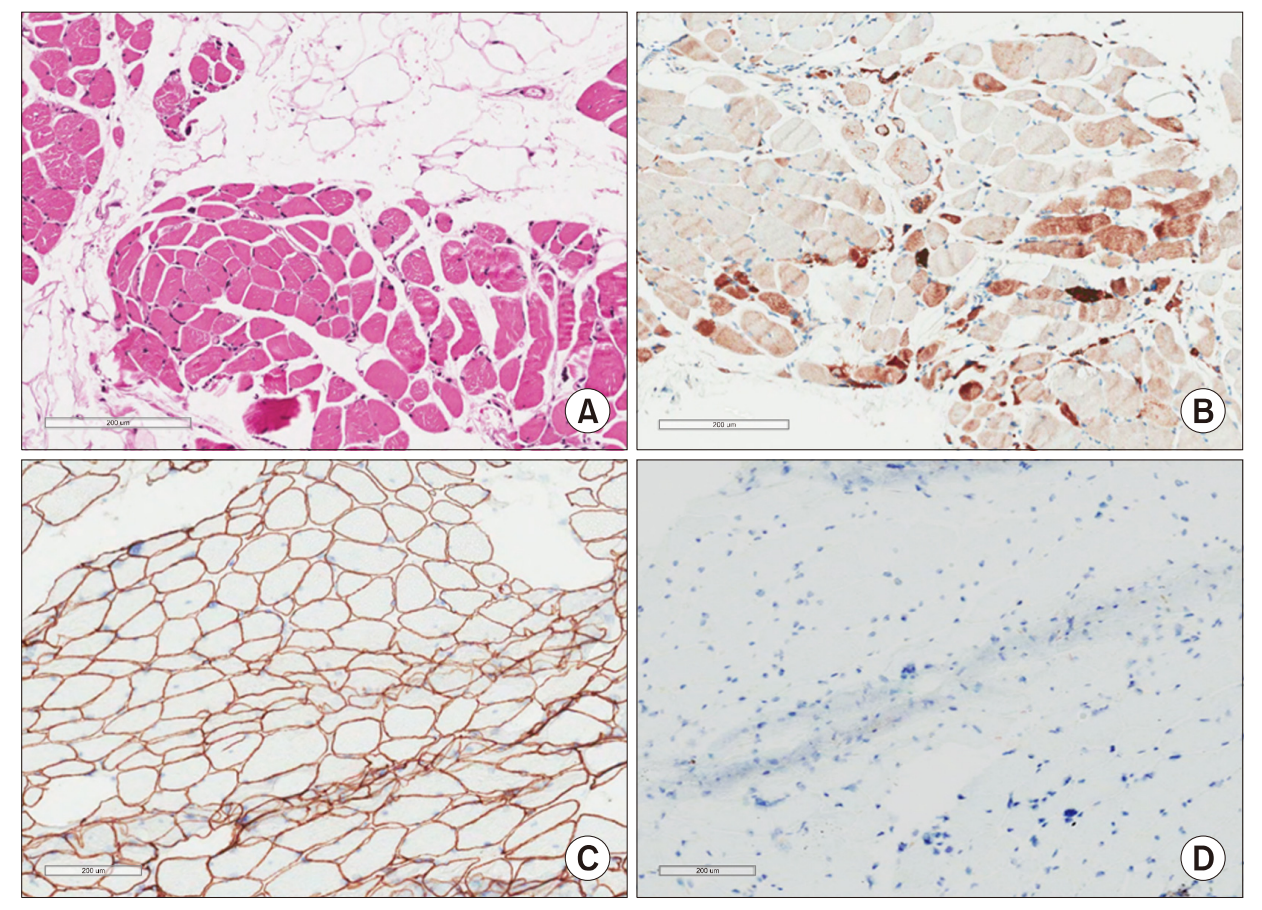

Figure 2. H\&E stain $(A)$ and immunohistochemistry $(B \sim D)$ of the muscle biopsy. (A) Myofibers show moderate size variation with degenerating cells with endomysial fibrofatty change $(\mathrm{H} \& \mathrm{E}$, $\times 200$ ). (B) Many degenerated myofibers show variation of size and positivity for CD56 (CD56 immunohistochemistry, $\times 100)$. Dystrophin (C) was robustly positive (Dystrophin 1 immunohistochemistry, $\times 200)$, yet dysferlin (D) was totally absent in the sarcolemmal membrane (Dysferlin immunohistochemistry, $\times 200)$.

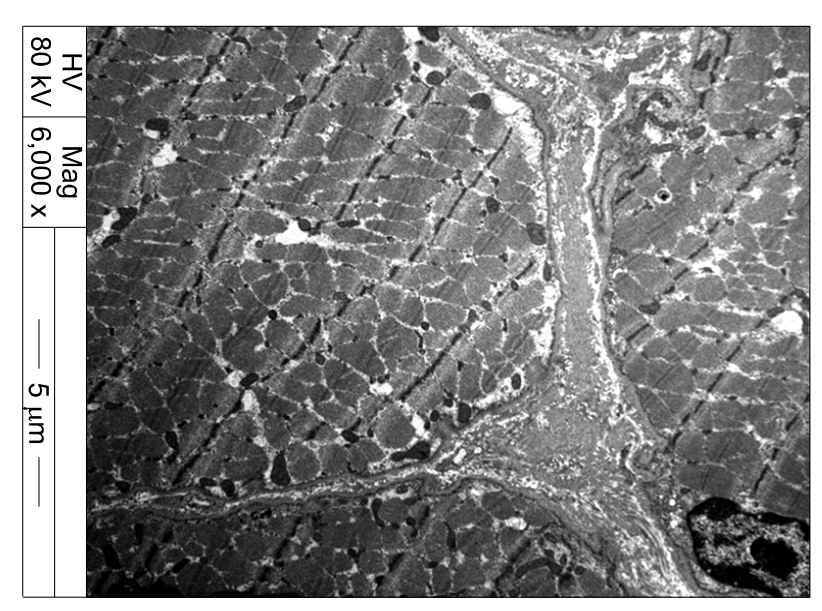

Figure 3. Electron microscopy of the muscle. Myofibers show size variation with mild atrophic change. Lay-down of collagen are present within the endomysium (Uranyl acetate and lead citrate, $\times 6,000$ ).

[6]. Sanger sequencing confirmed that the mutations segregated with the disease phenotype (Figure 4). A final diagnosis of LGMD2B was made, and all immunosuppressive agents were tapered off. The patient remained stable on physiotherapy.

The study was approved by the Institutional Review Board of Seoul Metropolitan Government-Seoul National University Boramae Medical Center (IRB No. 16-201761). Informed consent was obtained from the patient.

\section{DISCUSSION}

Our case presents a 48-year-old male with progressive proximal muscle weakness in the lower limbs, distinctive skin rashes, high CK levels, and a myopathic pattern in the EMG. He was initially diagnosed with DM, but showed poor treatment response to immunosuppressive agents. After dysferlin immunostaining of the muscle tissue and genetic analysis, a final diagnosis of LGMD2B was made.

Dysferlin, a protein encoded by the DYSF gene located on chromosome $2 \mathrm{p} 13$, plays a role in vesicle docking and fusion with the plasma membrane to repair sarcolemma disruption. Thus, mutations in the DYSF gene are responsible for a defective repair mechanism in the sarcolemma leading to muscular dystrophy: a LGMD2B affecting the proximal lower limbs at the onset, and a Miyoshi myopathy (MM) affecting posterior distal compartment of the legs [7]. Both LGMD2B and MM have similar features, however, muscle weakness with pelvic girdle distribution distinguishes between LGMD2B and MM. Interestingly, the type of DYSF gene mutation does not correlate with phenotypic features of dysferlinopathy and shows clinical heterogeneity even with the same mutation [8]. Patients with dysferlinopathy, especially LGMD2B, have symmetric proximal weakness, high CK levels and small polyphasic motor-unit potentials in the EMG, which may be confused with inflammatory myopathy. Nguyen et al. [4] 


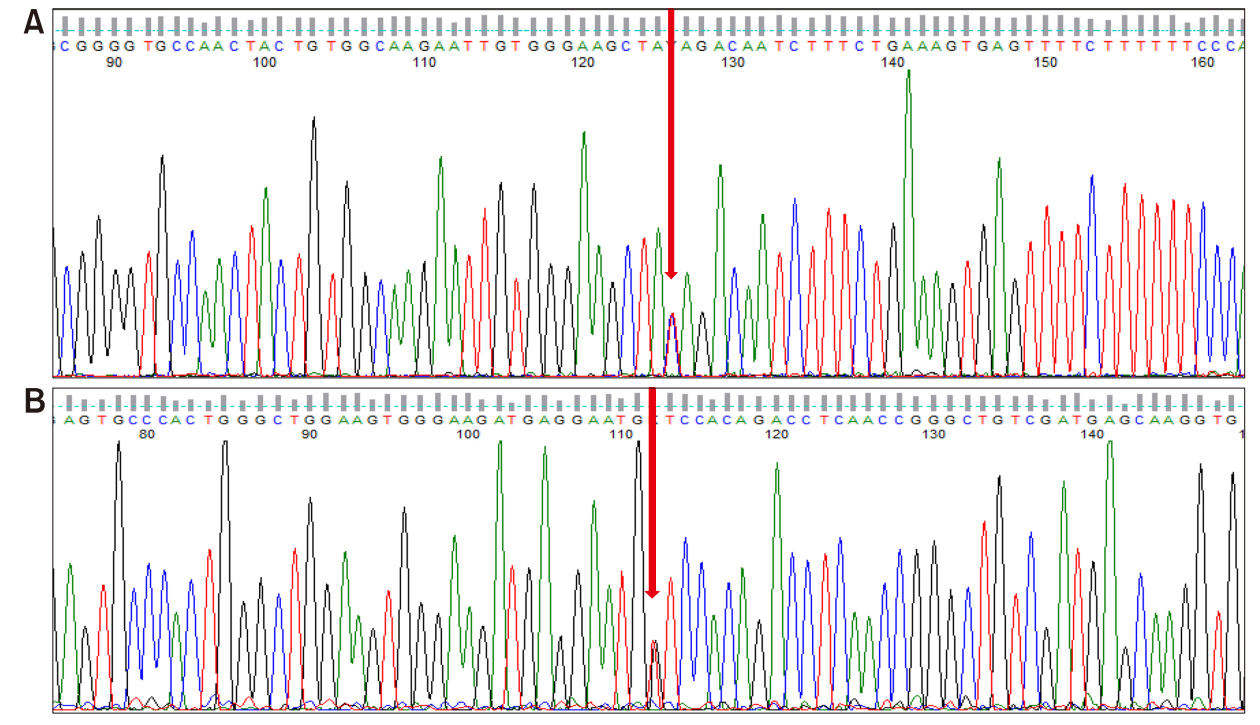

Figure 4. Sanger sequencing of the mutations in allele c. 2548 C $>$ T (p.Gln850*) (nonsense mutation) (A) and c.3051G $>$ T (p.Trp1017Cys) (missense mutation) (B) (arrows). reported that approximately $25 \%$ of patients with dysferlinopathy are initially misdiagnosed with inflammatory myopathy. Nevertheless, the two entities display several different clinical features. First, patients with dysferlinopathy commonly demonstrate inability to stand or walk on toes due to distal muscle involvement and atrophy of the gastrocnemius [9]. Second, the disease onset of dysferlinopathy usually occurs during early adulthood (typically 15 to 35 years), and patients may have a pre-clinical period with relatively high $\mathrm{CK}$ levels ranging from 1,000 to $40,000 \mathrm{IU} / \mathrm{L}[3,10]$. Third, treatment response to glucocorticoid and/or other immunosuppressive agents is far from adequate. The mainstay management of dysferlinopathy is exercise to maintain muscle strength [11]. Table 1 compares the characteristics of dysferlinopathy with those of inflammatory myopathies.

The precise distinction between dysferlinopathy and inflammatory myopathy is based on muscle biopsy and dysferlin immunostaining, which shows a significant reduction or absence of dysferlin protein in the sarcolemma in dysferlinopathy [12]. Interestingly, inflammatory cell infiltrates in muscles are often present in dysferlinopathy [13]. Inflammatory cell types in dysferlinopathy shows high CD4+/CD8 + T cell ratios predominantly in the perivascular region similar to DM. But unlike inflammatory myopathy, major histocompatibility complex class I expression is low and sarcolemmal membrane attack complex is deposited on non-necrotic fibers [5]. In our case, IMNM should also be considered since the patient was on statin therapy, but no muscle cell necrosis or macrophage infiltration was found in the biopsy specimen [14].
Our patient demonstrated several unusual features of dysferlinopathy that made the diagnosis difficult. First, age of onset was later in life compared with that presented in previous studies. Park et al. [15] reported that the age of onset varied from 9 to 36 years in Korean patients with dysferlinopathy. A Japanese study showed a wider range of age; 14 to 58 years [16]. Late-onset LGMD2B is associated with the homozygous mutation of the c.2997G $>$ T (p.Trp999Cys) allele in Japanese [16]. Two of 23 Korean patients with dysferlinopathy showed a heterozygous mutation in the c.2997G > T allele; one was a 41-year-old male with Miyoshi myopathy and the other was a 27-year-old female with LGMD2B [6]. The mutations in our patient and its association with a late-onset phenotype need to be further investigated. Second, psoriasis sporadically appeared in multiple regions in our patient along with muscle weakness. There is no report on the association between dysferlinopathy with psoriasis to date. It is sometimes challenging to distinguish between psoriasis and Gottron's papules seen in DM as the two often develop at similar anatomical sites.

\section{SUMMARY}

In summary, due to the clinical heterogeneity of dysferlinopathy and its overlapping features with inflammatory myopathy, it could sometimes be difficult to discern the two entities. Dysferlinopathy should be considered in patients with myopathy who have markedly high CK levels, calf muscle atrophy, and poor response to immunosuppressive therapy. Muscle dysferlin immunostaining 


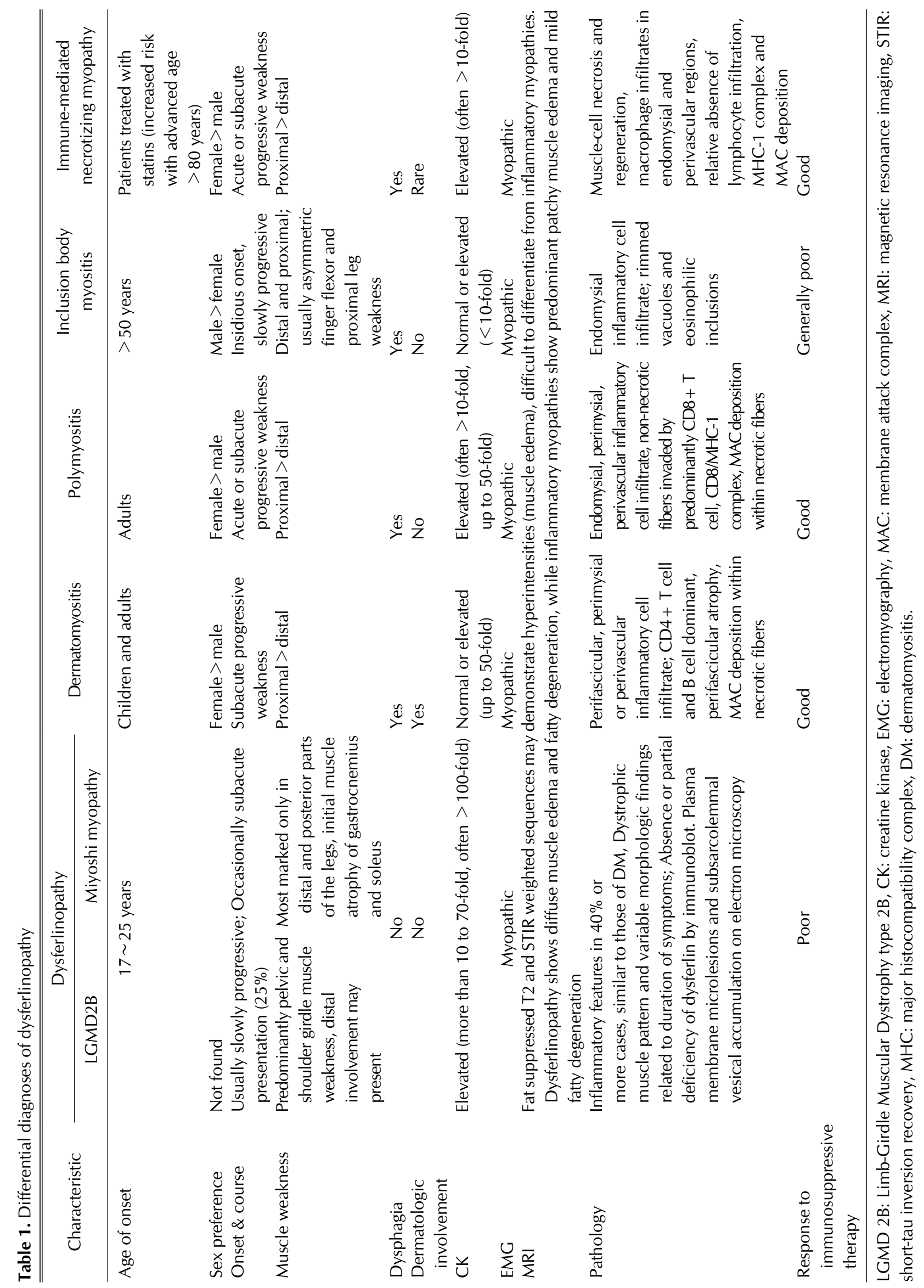


plus genetic analysis would be essential for its diagnosis.

\section{CONFLICT OF INTEREST}

No potential conflict of interest relevant to this article was reported.

\section{AUTHOR CONTRIBUTIONS}

J.S.O. and K.S. contributed to the study conception and design. Material preparation, data collection and analysis were performed by M.J.K. ${ }^{1}$, Y.O., Y.H.H., S.P., J.S.O., M.J.K. ${ }^{6}$ and J.C. The first draft of the manuscript was written by M.J.K. ${ }^{1}$ and all authors commented on previous versions of the manuscript. All authors read and approved the final manuscript.

\section{REFERENCES}

1. Dalakas MC. Inflammatory muscle diseases. N Engl J Med 2015;372:1734-47.

2. Bohan A, Peter JB. Polymyositis and dermatomyositis (first of two parts). N Engl J Med 1975;292:344-7.

3. Wicklund MP, Kissel JT. The limb-girdle muscular dystrophies. Neurol Clin 2014;32:729-49.

4. Nguyen K, Bassez G, Bernard R, Krahn M, Labelle V, Figarella-Branger D, et al. Dysferlin mutations in LGMD2B, Miyoshi myopathy, and atypical dysferlinopathies. Hum Mutat 2005;26:165.

5. Choi JH, Park YE, Kim SI, Kim JI, Lee CH, Park KH, et al. Differential immunohistological features of inflammatory myopathies and dysferlinopathy. J Korean Med Sci 2009;
24:1015-23

6. Park YE, Kim HS, Lee CH, Nam TS, Choi YC, Kim DS. Two common mutations (p.Gln832X and c.663+1G $>$ C) account for about a third of the DYSF mutations in Korean patients with dysferlinopathy. Neuromuscul Disord 2012;22: 505-10.

7. Bansal D, Miyake K, Vogel SS, Groh S, Chen CC, Williamson $\mathrm{R}$, et al. Defective membrane repair in dysferlin-deficient muscular dystrophy. Nature 2003;423:168-72.

8. Petersen JA, Kuntzer T, Fischer D, von der Hagen M, Huebner A, Kana V, et al. Dysferlinopathy in Switzerland: clinical phenotypes and potential founder effects. BMC Neurol 2015;15:182.

9. Tarnopolsky MA, Hatcher E, Shupak R. Genetic myopathies initially diagnosed and treated as inflammatory myopathy. Can J Neurol Sci 2016;43:381-4.

10. Klinge L, Aboumousa A, Eagle M, Hudson J, Sarkozy A, Vita $G$, et al. New aspects on patients affected by dysferlin deficient muscular dystrophy. J Neurol Neurosurg Psychiatry 2010;81:946-53.

11. Biondi O, Villemeur M, Marchand A, Chretien F, Bourg N, Gherardi RK, et al. Dual effects of exercise in dysferlinopathy. Am J Pathol 2013;182:2298-309.

12. Fanin M, Angelini C. Progress and challenges in diagnosis of dysferlinopathy. Muscle Nerve 2016;54:821-35.

13. Vinit J, Samson M Jr, Gaultier JB, Laquerriere A, Ollagnon E, Petiot $\mathrm{P}$, et al. Dysferlin deficiency treated like refractory polymyositis. Clin Rheumatol 2010;29:103-6.

14. Mammen AL. Statin-associated autoimmune myopathy. N Engl J Med 2016;374:664-9.

15. Park HJ, Hong JM, Suh GI, Shin HY, Kim SM, Sunwoo IN, et al. Heterogeneous characteristics of Korean patients with dysferlinopathy. J Korean Med Sci 2012;27:423-9.

16. Takahashi T, Aoki M, Suzuki N, Tateyama M, Yaginuma C, Sato $\mathrm{H}$, et al. Clinical features and a mutation with late onset of limb girdle muscular dystrophy 2B. J Neurol Neurosurg Psychiatry 2013;84:433-40. 